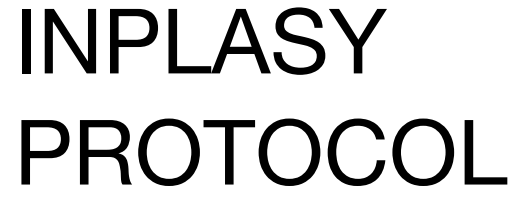

To cite: Ibeneme et al. Effect of physical exercise on lower limb muscular function, ambulatory function and lower limb fracture healing in people living with HIV and AIDS: a

systematic review. Inplasy protocol 202090059. doi: 10.37766/inplasy2020.9.0059

Received: 14 September 2020

Published: 14 September 2020

Corresponding author:

Gloria Amarachi Eneh

enehgloriaamara@gmail.com

Author Affiliation:

University of Nigeria Nsukka

Support: None.

Review Stage at time of this submission: Preliminary searches.

Conflicts of interest: University of Nigeria Enugu Campus.

\section{Effect of physical exercise on lower limb muscular function, ambulatory function and lower limb fracture healing in people living with HIV and AIDS: a systematic review}

Ibeneme, SC1; Eneh, GA2; Anieto, EM³3.

Review question / Objective: What effect does exercise have on muscle strength of people living with HIV and AIDS (PLWHA)? What effect does exercise have on ambulatory function of PLWHA? What effect does exercise have on fracture healing in PLWHA? What are the FITT parameters that could improve muscular strength, gait outcomes and fracture healing in PLWHA?

Condition being studied: Fragility fracture and poor gait performance are the common musculoskeletal complications of HIV. HIV and the use of highly active antiretroviral therapy (HAART) have been associated with reduction in bone mineral density (Mondy et al., 2003; Mondy \& Tebas, 2003; Singh, 2006) as well as, delayed and nonunion of fractures (Richardson et al., 2008). Disruption of the normal bone metabolism common in PLWHA affects the bone mineral density which further predispose PLWHA to fragility fracture (Jacobson et al.,2010). Fragility fracture is $\mathbf{3 0 - 7 0 \%}$ higher in PLWHA when compared to sero-negative individuals (Triant et al., 2008; Womack et al., 2010) and it's usually a challenge as a result of high infection rate and osteonecrosis in PLWHA. Consequently, healing is delayed or complicated with non-union. More so, ambulation a crucial aspect of physical function is limited in PLWHA (Berner et al., 2017), however, many factors are predictors of gait performance including muscular function. The high incidence of wasting syndrome in HIV has been associated with reduced muscular strength and functional performance among PLWHA (coats 2002). Wasting syndrome is simply reduction in lean body mass or fat free mass which skeletal muscles contribute about $50 \%$ of it.

INPLASY registration number: This protocol was registered with the International Platform of Registered Systematic Review and Meta-Analysis Protocols (INPLASY) on 14 September 2020 and was last updated on 14 September 2020 (registration number INPLASY202090059).

\section{INTRODUCTION}

Review question / Objective: What effect does exercise have on muscle strength of people living with HIV and AIDS (PLWHA)? What effect does exercise have on ambulatory function of PLWHA? What effect does exercise have on fracture 
healing in PLWHA? What are the FITT parameters that could improve muscular strength, gait outcomes and fracture healing in PLWHA?

Rationale: Muscle wasting (Richert et al., 2011),, poor gait performance (Berner et al 2017) and fragility fractures (Triant et al., 2008; Womack et al., 2010) with delayed healing or non-union among others are the common musculoskeletal complications of HIV. Considering that adverse effect of HIV treatments predispose PLWHA to more health problems (Mondy et al., 2003; Mondy \& Tebas, 2003; Singh, 2006), to reduce the effect of drug toxicity there is need for nonpharmacological approaches like exercise to mitigate these complications. Exercise seems to be beneficial in mitigating the health complications caused by HIV and the side effects of HAART (Hand et al., 2009), thus improving the health outcomes of PLWHA (Botros et al., 2012). Various systematic reviews have reported the plausible effect of physical activity/exercise on outcomes such as, inflammatory markers, quality of life, cardiopulmonary fitness of PLWHA (Ibeneme et al 2019; O'Brien et al., 2017; Zech et al., 2018). However, there is paucity of reviews on the impact of exercise on gait and fracture healing among PLWHA. Therefore, this review is aimed at evaluating the effect of physical exercise on muscular function, ambulatory function and fracture healing in PLWHA. This review will also provide evidence on the frequency, intensity, time, and type of exercise intervention (FITT) that improve muscular strength, gait outcomes and fracture healing in PLWHA.

Condition being studied: Fragility fracture and poor gait performance are the common musculoskeletal complications of HIV. HIV and the use of highly active antiretroviral therapy (HAART) have been associated with reduction in bone mineral density (Mondy et al., 2003; Mondy \& Tebas, 2003; Singh, 2006) as well as, delayed and nonunion of fractures (Richardson et al., 2008). Disruption of the normal bone metabolism common in PLWHA affects the bone mineral density which further predispose PLWHA to fragility fracture
(Jacobson et al.,2010). Fragility fracture is $30-70 \%$ higher in PLWHA when compared to sero-negative individuals (Triant et al., 2008; Womack et al., 2010) and it's usually a challenge as a result of high infection rate and osteonecrosis in PLWHA. Consequently, healing is delayed or complicated with non-union. More so, ambulation a crucial aspect of physical function is limited in PLWHA (Berner et al., 2017), however, many factors are predictors of gait performance including muscular function. The high incidence of wasting syndrome in HIV has been associated with reduced muscular strength and functional performance among PLWHA (coats 2002). Wasting syndrome is simply reduction in lean body mass or fat free mass which skeletal muscles contribute about $50 \%$ of it.

\section{METHODS}

Search strategy: The search strategy was developed by combinations of search terms from medical subject headings (MeSH) and keywords with a combination of Boolean logic. Below is the search strategy; ((HIV OR AIDS OR RNA virus OR lentivirus OR AIDS viruses) AND (exercise OR physical activity OR sports OR physical fitness OR training OR physical exertion OR motor activities)) AND (muscle performance OR muscle strength OR muscle hypotonia OR sarcopenia OR floppy muscle OR muscle flaccidity) Filters: Randomized Controlled Trial ((HIV OR AIDS OR RNA virus OR lentivirus OR AIDS viruses) AND (exercise OR physical activity OR sports OR physical fitness OR training OR physical exertion OR motor activities)) AND (fracture OR bone fracture OR femoral fracture OR hip fracture OR avulsion) Filters: Randomized Controlled Trial ((HIV OR AIDS OR RNA virus OR lentivirus OR AIDS viruses) AND (exercise OR physical activity OR sports OR physical fitness OR training OR physical exertion OR motor activities)) AND (walking OR ambulation OR walking speed OR walk test OR functional mobility OR gait OR walking aid OR movement OR balance OR locomotion) Filters: Randomized Controlled Trial. 
Participant or population: Adult human participants aged $\geq 18$ years living with HIV.

Intervention: Physical exercise or exercise interventions, which include aerobic exercises, resistance exercise or both.

\section{Comparator: Non- exercise group}

Study designs to be included: Any type of Randomized controlled trials (RCTs) that compared physical exercise training with non-exercise training or with any other therapeutic management. Authors of a studies with vague methodology will be contacted by email to offer clarifications where necessary. And studies with poor methodological quality will be excluded.

Eligibility criteria: Inclusion criteria Type of studies: This review includes only randomized control trials published in English language which assessed the effects of exercise trainings on lower limb muscular functions, ambulatory function and fracture healing in PLWHA. Participants: The review will include only RCTs involving adults ( $>18$ years) living with HIV and AIDS, who are either on HAART or HAART-naïve with no specific limitation on settings. Control: studies that compared the effectiveness of exercise training on muscular function, ambulatory function and fracture healing to any other treatment option like sham or placebo treatment etc. will be included in this review. Outcomes: studies reported muscular function, ambulatory function and fracture healing or any other outcome that are predictors of the listed primary outcomes in PLWHA will be considered. Exclusion criteria Studies without physical exercise component or studies that did not report the outcome of interest in this review or studies with vague methodology, narratives, reviews (scooping and systematic reviews), opinion papers, letters and/or publications without primary data will be excluded. Editorials and newspaper articles will be excluded. Also studies that were not published in English language.

Information sources: Relevant studies will be retrieved by searching the following
Databases AMED, CINAHL the Cochrane Library, Ovid, MEDLINE, Psyclnfo and PEDro with no limit on date of publication. Further search will be conducted on the reference list of retrieved studies. This review will include only studies published in English language and RCTs that gave exercise interventions. An elaborate search to identify relevant studies will be done in two stages including; (i) the search of the bibliographic database and grey literature, and (ii) the selection of studies for inclusion based on eligibility criteria.

Main outcome(s): Primary outcomes of interest are the following: lower limb muscular function, ambulatory function and fracture healing. Although studies with these outcomes alongside other outcomes will not be excluded.

Additional outcome(s): While secondary outcomes include muscle bulk/mass, muscle strength, gait speed, cadence, 6MWT, balance, bone turnover, callus formation, osteoblastic and osteoclastic activity.

Data management: The retrieved articles after the literature search will be exported into RefWorksTM to check for duplicates. The bibliographic records will be exported from RefWorksTM into Microsoft Excel 2007 where articles selection for inclusion into the study will be based on the eligibility criteria.

Quality assessment / Risk of bias analysis: Two reviewers will independently assess the methodological quality and the Cochrane Collaboration's tool for risk of bias assessment will be used to assess risk of bias in selected articles. This includes reference to sequence generation, allocation concealment, blinding, incomplete outcome data (dropouts and withdrawals) and selective outcome reporting.

Strategy of data synthesis: Meta-analysis will be conducted where feasible. The impacts of physical exercise on lower limb muscular function, ambulatory function and lower limb fracture healing in HIV/AIDS 
patients will be determined by an assessment of all the quantitative study outcomes which have analyzed the effects of these interventions. Analysis and presentation of results will be on a table and validated statistical methods will be used to evaluate the different variables.

Subgroup analysis: In the case of having a mix of heterogeneous and homogenous studies, the homogenous will be pooled together for a subgroup analysis.

Sensibility analysis: The robustness of the meta-analysis will be assessed through sensitivity analysis, low quality trials and small sample trials will be excluded in order to evaluate the impact of trial quality on efficacy estimates. Furthermore, a second meta-analysis will be conducted based on the outcome of the sensitivity analysis.

Language: Only RCTs published in English Language.

Country(ies) involved: Nigeria and South Africa.

Other relevant information: None.

Keywords: Fracture healing, ambulatory function, HIV and AIDS, muscle strength.

Dissemination plans: GRADE Evidence Table and Descriptive Evidence Table will be drafted and submitted to the WHO Secretariat as stipulated in the Procedures for the Retrieval of Evidence and Summary of Evidence. Review protocol will be registered and published with INPLASY international platform of registered systematic reviews and meta-analysis protocol Also, a manuscript will be submitted to a peer-reviewed journal for publication. Conference presentations on the study will be made where necessary.

Contributions of each author:

Author 1 - Dr. Samuel Chidi Ibeneme Conceived the protocol; Designed the protocol; Reading, editing and approving of the final draft of the manuscript.
Author 2 - Gloria Amarachi Eneh Conceived the protocol; Designed the protocol; Developed the search strategy; Reading and editing the approved manuscript.

Author 3 - Ebuka Miracle Anieto - Designed the protocol Developed the search strategy Reading, editing and approving of the final draft of the manuscript. 